\title{
Effets comparés de la substitution partielle du tourteau de soja par la farine d'asticots séchés dans des rations alimentaires sur les performances zootechniques des poulets de chair à Ngaoundéré au Cameroun
}

\author{
A. Mebanga SASSA*, Éric J. MBALLA et Mamoudou ABDOULMOUMINI \\ Ecole des Sciences et de Médecine Vétérinaire, Département de Production Animale, Université de \\ Ngaoundéré, B.P. 268 Ngaoundéré, Cameroun. \\ *Auteur correspondant, E-mail: mebengasaaristide@gmail.com
}

\begin{tabular}{lll}
\hline Received: 01-07-2021 & Accepted: 26-11-2021 & Published: 31-12-2021 \\
\hline
\end{tabular}

\section{RESUME}

Les asticots sont une source importante de protéines animales faiblement valorisées dans l'alimentation des poulets de chair. L'étude a été menée dans le but d'analyser et d'évaluer les effets comparés de l'utilisation du tourteau de soja et la farine d'asticots dans les rations alimentaires de démarrage et de croissance sur les performances zootechniques des poulets de chair à Ngaoundéré au Cameroun. Le gain de poids pour les périodes de démarrage et de fin de croissance était élevé chez les poulets nourris avec des rations contenant la farine d'asticots séchés comparé aux sujets témoins, nourris avec la ration contenant uniquement du tourteau de soja. Pendant toute la durée des essais, on n'a pas observé une différence significative $(p>0,05)$ entre les différentes rations pour l'indice de consommation et la consommation des aliments. Néanmoins, une meilleure utilisation des aliments était constatée chez les sujets nourris à la ration à base de farine d'asticots séchés. Les mortalités ont été relativement élevées au démarrage. D'un point de vue technique et économique, la farine d'asticots séchés pourrait mieux remplacer le tourteau de soja à un faible taux (10\%) dans les aliments des poulets de chair et être recommandée en aviculture.

(C) 2021 International Formulae Group. All rights reserved.

Mots clés : Asticots, performances zootechniques, poulet de chair, tourteau de soja.

\section{Comparative effects of partial substitution of soybean meal by dried maggot flour in feed rations on the zootechnical performances of broilers in Ngaoundere in Cameroon}

\begin{abstract}
Maggots are an important source of low value animal protein in the diet of broilers. The study has been carried out with the aim of analyzing and evaluating the comparative effects of the use of soybean meal and dried maggot meal in starter and growth feed rations on the zootechnical performances of chickens from flesh in Ngaoundere in Cameroon. Weight gain for the start and end of growth periods was high in chickens fed rations containing dried maggot meal compared to controls fed rations containing only soybean meal. During the entire duration of the tests, a significant difference $(p>0,05)$ between the different rations was not observed for the
\end{abstract}


consumption index and the food consumption. However, improved feed utilization has been observed in subjects fed the dried maggot meal ration. Mortalities were relatively high at start-up. From technical and economic point of view, the meal of dried maggot could better replace soybean meal at a rate of $10 \%$ in the feed of broilers and be recommended in poultry farming.

(C) 2021 International Formulae Group. All rights reserved.

Keywords: Maggots, zootechnical performances, broilers, soybean meal.

\section{INTRODUCTION}

Le développement de l'aviculture en Afrique en général, et au Cameroun en particulier est freiné en grande partie par l'insuffisance des ressources alimentaires, et surtout la carence des rations alimentaires en protéines qui ne couvre pas toujours les besoins des animaux (Loa, 2000). Cette situation est aggravée par le coût élevé des aliments, l'insuffisance des devises destinées à l'importation des ingrédients et la compétition entre l'Homme et l'animal pour les mêmes ressources alimentaires (Minepat, 2013). Aussi, la production d'aliments à moindre coût destinés à l'alimentation de la volaille est difficile. Pour baisser le coût de production en aviculture, il importe de trouver des solutions alternatives locales moins coûteuses pour pallier les coûts élevés des matières premières (Mpoame, 2004). L'une des alternatives est l'incorporation des asticots dans l'alimentation de la volaille. Les asticots sont faciles à produire et peuvent être incorporés comme source de protéines dans l'alimentation des animaux monogastriques tels que la volaille et le poisson (Kenis, 2014). En outre, les asticots sont des bio-dégradeurs des ordures ménagères dont la gestion constitue une préoccupation environnementale majeure en Afrique (Pomalegni, 2020). De plus, l'asticot présente l'avantage qu'en Afrique subsaharienne, sa production en grande quantité est possible à partir d'une large gamme d'ordures ménagères, de sous-produits agricoles et de déchets d'origine animale (Bouafou, 2011). Des résultats satisfaisants en matière de l'évolution pondérale des oiseaux ont été obtenus avec l'utilisation des asticots sous diverses formes dans l'alimentation de la volaille (Loa, 2000), mais au Cameroun, très peu de données existent en ce qui concerne les performances zootechniques des poulets de chair soumis à des rations alimentaires à base d'asticots. C'est dans cette perspective que cette étude s'est proposée d'analyser et d'évaluer l'influence d'une alimentation à base d'asticots sur les performances zootechniques des poulets de chair à Ngaoundéré au Cameroun.

\section{MATERIEL ET METHODES}

\section{Milieu et période d'étude}

L'étude s'est déroulée en zone périurbaine, dans la localité de Dang située dans le $3^{\text {ème }}$ arrondissement de la ville de Ngaoundéré, chef-lieu de la région de l'Adamaoua (Figure 1), plus précisément dans une ferme aménagée sur le site de production des larves d'asticots. La ville de Ngaoundéré couvre une superficie de $63701 \mathrm{~km}^{2}$ et est située à une latitude de $7^{\circ} 19$ Nord, une longitude de $13^{\circ} 35$ Est et à une altitude moyenne de $1100 \mathrm{~m}$. La zone est caractérisée par un climat tropical de type soudano-guinéen avec une saison sèche allant de novembre à mars et une saison pluvieuse allant d'avril à octobre. Les températures sont relativement stables soit $22^{\circ} \mathrm{C}$ en moyenne avec un maximum de $34,6^{\circ} \mathrm{C}$ en mai et un minimum de $9,9^{\circ} \mathrm{C}$ en janvier. Les pluies y sont abondantes avec une moyenne de $1539,3 \mathrm{~mm} / \mathrm{an}$. La végétation est constituée principalement des savanes arborées et arbustives. La population est estimée à 1080500 habitants avec une densité de 17 habitants $/ \mathrm{km}^{2}$ (Minepat, 2013). La population est cosmopolite avec pour principales activités : l'agriculture, l'élevage, la pêche, l'apiculture et le commerce. Les essais ont duré 8 semaines, du 10 octobre au 25 novembre 2020.

\section{Choix du milieu d'expérimentation}

La ferme située dans la localité de Dang a été choisie en fonction de son accessibilité, de 
la disponibilité de l'éleveur à abriter nos travaux, et de la proximité avec la structure de ravitaillement en asticots.

\section{Matériel animal et dispositif expérimental}

L'étude a porté sur une bande de 75 poussins d'un jour, non sexés, de souche Cobb 500 , d'un poids moyen de $38 \mathrm{~g}$, achetés à la société avicole ALIVET. Les essais ont été conduits dans une ferme composée de deux blocs de poulaillers disposés face à face. Ces blocs ont été compartimentés chacun en loge de $16 \mathrm{~m}^{2}$ bien aérés, abritant chaque lot. L'aération et l'éclairage étaient assurés par des ouvertures grillagées sur les murs à une hauteur de $1,5 \mathrm{~m}$ du sol. Des abreuvoirs et des mangeoires ont été installés dans les poulaillers. Dès leur réception à la ferme, les poussins ont reçu le vaccin contre la maladie de Newcastle par trempage oculonasal. Un antistress leur a été apporté dans l'eau de boisson. Ils ont subi un plan prophylactique minimal (couverture vaccinale, déparasitage interne et externe, antibiothérapie et antistress). Le Tableau 1 décrit le programme de prophylaxie appliqué tel que prescrit par la société ALIVET.

\section{Mise en lots des animaux et alimentation}

Les 75 poussins ont été répartis, dès leur réception, de manière aléatoire, en trois lots de 25 sujets. Une transition alimentaire de 5 jours a été observée avant le démarrage effectif des essais. Par la suite, trois rations alimentaires ont été constituées comme suit :

- Une ration témoin $\mathrm{R} 1$ avec $0 \%$ de farine d'asticots séchés dans la composition ;

- Une ration expérimentale R2 dont le taux de substitution du tourteau de soja par la farine d'asticots séchés est de $5 \%$ dans la composition ;

- Une ration expérimentale R3 dont le taux de substitution du tourteau de soja par la farine d'asticots séchés est de $10 \%$ dans la composition.

Les ingrédients alimentaires qui ont servi à formuler les rations étaient le maïs grain, le son de blé, le tourteau de soja, le tourteau de palmiste, la farine d'os, la farine d'asticots et le sel de cuisine.
L'aliment a été servi à volonté deux fois par jour à $7 \mathrm{~h} 30$ et $16 \mathrm{~h} 00$, de l'eau potable a été mise sans restriction à la disposition de tous les animaux dans des abreuvoirs siphoïdes. L'aliment non consommé a été chaque fois récupéré et pesé. Les essais étaient subdivisés en une phase de démarrage et une phase de croissance d'une durée respective de 4 semaines. Pour la phase de démarrage, trois rations de même composition centésimale ont été confectionnées (Tableau 2), la seule différence entre ces rations se situait au niveau du taux de substitution du tourteau de soja par la farine d'asticots séchés. A l'issue de la phase de démarrage, les animaux ont été nourris avec une ration de croissance dont le taux d'incorporation de protéine animale a été réduit de moitié (Tableau 3).

La formulation des aliments a été élaborée en tenant compte d'une part des besoins nutritifs des poulets de chair et d'autre part de la disponibilité des matières premières dans la zone d'étude. Les aliments ont été formulés pour présenter des teneurs isoprotéiques et iso-énergétiques. Les teneurs en nutriments (Tableau 4) ont été calculées selon les méthodes officielles approuvées par le laboratoire du Génie des Procédés de l'Ecole Nationale Supérieure des Sciences AgroIndustrielles (ENSAI) de l'Université de Ngaoundéré au Cameroun.

\section{Obtention de la Farine d'asticots séchés}

Les asticots utilisés pour obtenir la farine ont été produits à partir des fientes des poules pondeuses. La fiente une fois collectée dans des bacs en plastic d'une contenance de 100 litres, a été exposée à l'air libre, à l'ombre des arbres. Le contenu des bacs a été aspergé d'eau une fois par jour afin d'éviter l'assèchement du substrat et accélérer par la même occasion sa décomposition. L'odeur dégagée par la décomposition du substrat a permis d'attirer des mouches domestiques de l'espèce Musca domestica qui sont venues y déposer des œufs. Après 24 heures d'ensemencement, les bacs sont recouverts 
d'une moustiquaire pour éviter des ensemencements tardifs et s'assurer ainsi de l'homogénéité des larves produites. La récolte des larves suffisamment développées a été effectuée 5 jours après le début de l'ensemencement. Les asticots recueillis ont été d'abord rincés à l'eau froide. La masse d'asticots obtenus a été laissée égouttée quelques minutes, puis étalée finement sur des tôles galvanisées ondulées pour être séchée au soleil. Un total de 6 jours d'exposition à raison de 7 heures d'ensoleillement intense par jour a été nécessaire pour obtenir un taux de déshydratation suffisant pour faciliter la mouture dans un moulin à maïs.

\section{Paramètres mesurés}

Les poussins ont été pesés dès le premier jour en lot afin d'avoir une idée sur leur poids moyen. Les pesées ont par la suite été hebdomadaires et individuelles à partir de la deuxième semaine; ce qui a permis l'estimation de l'évolution pondérale. Les quantités d'aliments consommés (servis et refus) ont été relevées par pesée quotidienne afin d'évaluer la consommation alimentaire. Le calcul de ces deux paramètres (croissance pondérale et consommation alimentaire) a permis l'estimation de l'indice de consommation. Les mortalités ont également été évaluées. Les mesures de poids des animaux et des aliments ont été effectuées à l'aide d'une balance électronique de précision $1 \mathrm{mg}$, une balance mécanique de précision $50 \mathrm{~g}$ et un peson à ressort de précision $10 \mathrm{~g}$. Un thermomètre était également placé dans le poulailler pour la mesure de la température ambiante.

\section{Analyse statistique}

Les données collectées au cours de l'étude ont concerné essentiellement l'analyse bromatologique de la farine d'asticots séchés et des différentes rations alimentaires, l'ingestibilité alimentaire, le taux de mortalité de même que les gains moyens quotidiens des poulets de chair. L'analyse statistique a été réalisée à l'aide du logiciel permettant les comparaisons nécessaires (test de t-student). Les effets des rations alimentaires sur les différents paramètres étudiés, ont été estimés par l'analyse de variance au seuil de 5\% à l'aide du logiciel SAS (Statistical Analysis System, version 9.2).

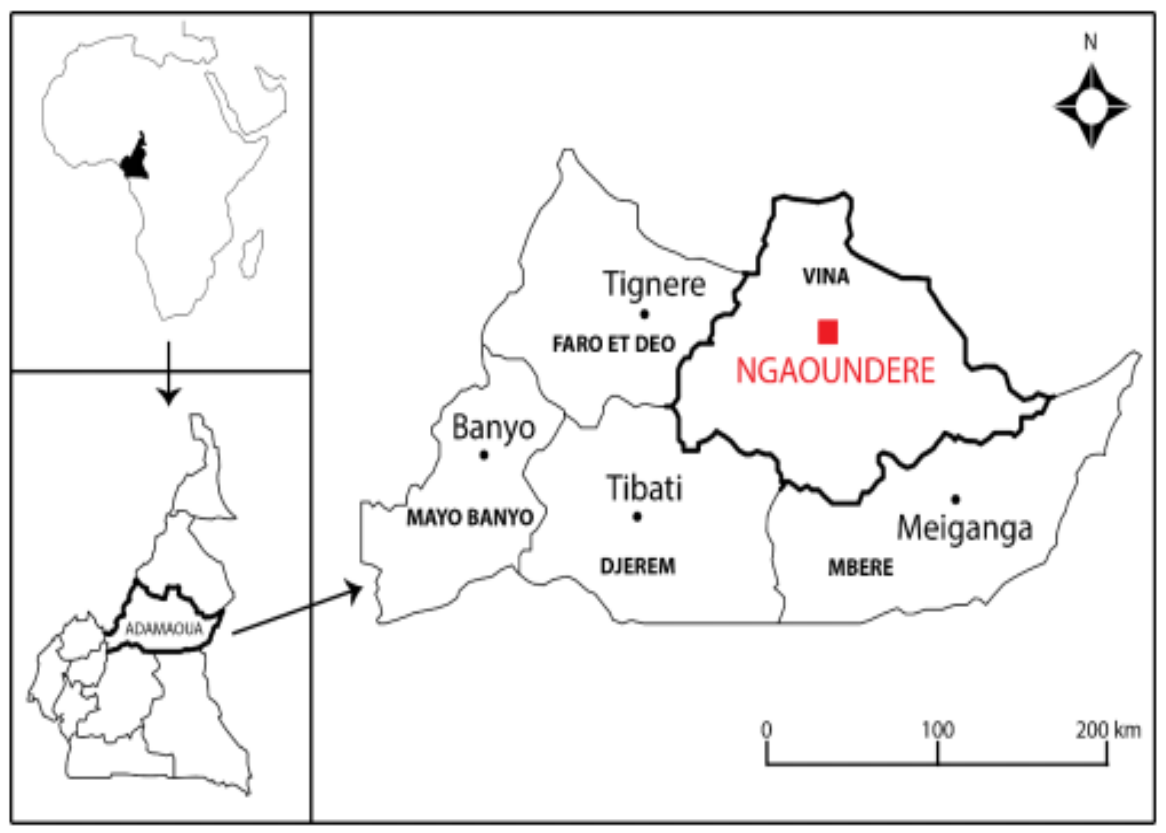

Figure 1: Zone d'étude. 
Tableau 1: Programme de prophylaxie appliqué durant les essais.

\begin{tabular}{|c|c|c|}
\hline $\begin{array}{l}\begin{array}{l}\text { Période } \\
\text { (jours) }\end{array} \\
\text { (jous }\end{array}$ & Produits & Intérêts \\
\hline \multirow[t]{2}{*}{$1^{\text {er }}$ au $3^{\text {ème }}$} & Vitamine C & $\begin{array}{l}\text { Antistress et augmente la résistance } \\
\text { des animaux }\end{array}$ \\
\hline & Vaccin Hitchner B1 & Vaccination contre Newcastle \\
\hline $3^{\text {ème }}$ au $5^{\text {ème }}$ & Tétracolivit & Prévention pos-vaccinale et antistress \\
\hline $12^{\text {ème }}$ au $16^{\text {ème }}$ & Vitamine A,D,E & $\begin{array}{l}\text { Stimule la croissance, fixe le } \mathrm{Ca} \text { et le } \mathrm{P} \text {, } \\
\text { favorise la formation et la contraction des } \\
\text { muscles }\end{array}$ \\
\hline $18^{\text {ème }}$ au $22^{\text {ème }}$ & Lobcox & Prévention contre la coccidiose \\
\hline $25^{\text {ème }}$ & Rappel Hitchner & Prévention contre Newcastle \\
\hline $35^{\text {ème }}$ & Lobcox & Rappel du coccidiostatique \\
\hline $42^{\text {ème }}$ & Tétracolivit & Antistress \\
\hline $42^{\text {ème }}$ au $48^{\text {ème }}$ & Vitamine A + oligo-éléments & Prévention contre l'encéphalomalacie \\
\hline $51^{\text {ème }}$ & Lobcox & Coccidiostatique pour période de finition \\
\hline
\end{tabular}

Tableau 2 : Composition centésimale des rations alimentaires des poulets de chair pendant la période de démarrage.

\begin{tabular}{lccc}
\hline Ingrédients (\%) & \multicolumn{3}{c}{ Rations démarrage } \\
& $\mathbf{R 1}$ & $\mathbf{R 2}$ & $\mathbf{R 3}$ \\
\hline Maïs gra & 50 & 50 & 50 \\
Son de blé & 20 & 20 & 20 \\
Tourteau de palmiste & 10 & 10 & 10 \\
Farine d'os & 04,5 & 04,5 & 04,5 \\
Sel de cuisine & 00,5 & 00,5 & 00,5 \\
Tourteau de soja & 15 & 10 & 05 \\
Farine d'asticots séchés & 00 & 05 & 10 \\
\hline Total & 100 & 100 & 100 \\
\hline
\end{tabular}

Tableau 3 : Composition centésimale des rations alimentaires des poulets de chair pendant la période de croissance.

\begin{tabular}{lccc}
\hline Ingrédients (\%) & R4 & Rations croissance \\
& 50 & 50 & R6 \\
\hline Maïs grain & 20 & 20 & 50 \\
Son de blé & 17 & 17 & 20 \\
Tourteau de palmiste & 05 & 05 & 05 \\
Farine d'os & 00,5 & 00,5 & 00,5 \\
Sel de cuisine & 07,5 & 05 & 02,5 \\
Tourteau de soja & 00 & 02,5 & 05 \\
Farine d'asticots séchés & 100 & 100 & 100 \\
\hline Total & & & \\
\hline
\end{tabular}


Tableau 4 : Composition moyenne des autres matières (en $\mathrm{g} / \mathrm{kg}$ et en $\mathrm{Kcal} / \mathrm{kg}$ pour EM) entrant dans la composition des rations utilisées au cours de l'essai.

\begin{tabular}{llllccc}
\hline Nutriments & Maïs & $\begin{array}{l}\text { Son de } \\
\text { blé }\end{array}$ & $\begin{array}{l}\text { Tourteau } \\
\text { de soja }\end{array}$ & $\begin{array}{c}\text { Tourteau } \\
\text { de palmiste }\end{array}$ & Farine d'os & Sel \\
\hline Matière sèche & 86,3 & 86,6 & 88,09 & 90,6 & 98 & - \\
Protéines brutes 07,6 & 14,9 & 51,52 & 16,6 & - & - \\
Cellulose brute 02,3 & 09,3 & 06,25 & 18,7 & - & - \\
Matière grasse & 03,6 & 04,5 & 01,98 & 02,9 & - & - \\
Cendres brutes 00,5 & 00,1 & 07,13 & 00,4 & 94,7 & 99,8 \\
Calcium & 04,0 & 01,2 & 00,34 & 02,7 & 34,4 & 04,3 \\
Phosphore & 02,5 & 09,0 & 00,73 & 05,5 & 15,25 & - \\
Sodium & 00,3 & 00,13 & 00,2 & 00,16 & - & 07 \\
Potassium & 03,1 & 11,2 & 02,4 & 06,7 & - & 01,4 \\
Magnésium & 01,0 & 02,7 & 00,33 & 03,1 & - & 00,2 \\
Lignine & 00,5 & 03,4 & 00,66 & 12,8 & - & - \\
Amidon & 59,3 & 21,6 & 05,27 & 00,5 & - & - \\
E.M. & 3840 & 3990 & 3500 & 4080 & & - \\
\hline
\end{tabular}

\section{RESULTATS}

\section{Composition chimique de la farine d'asticots} séchés et du tourteau de soja

La composition chimique de la farine d'asticots séchés et du tourteau de soja obtenue dans le laboratoire de recherche du département de Génie des Procédés de l'ENSAI a été présentée dans le Tableau 5. Il ressort des analyses chimiques effectuées (Tableau 5), que ces derniers sont particulièrement riches en Matière Azotée Totale (MAT) et en Matière Grasse (MG). Les valeurs pour ces éléments étaient respectivement de 59,64\% de MS et de $22,25 \%$ de MS pour la farine d'asticots séchés puis de $51,52 \%$ de MS et 1,98\% de MS pour le tourteau de soja. Cependant, leurs teneurs en minéraux étaient faibles.

\section{Evolution des effectifs et taux de mortalité des poulets de chair}

L'évolution des effectifs au cours de l'expérimentation est présentée dans le Tableau 6.

Le taux de mortalité au cours des essais était de $13,34 \%$. Le taux de mortalité des poulets soumis à la ration témoin R1 formulée avec exclusivement du tourteau de soja a été de $20 \%$ à l'issue de la période de démarrage. Ce taux s'est révélé deux fois plus élevé $(\mathrm{p}<0,05)$ que celui des poulets de chair nourris avec une ration R3 formulée avec un taux d'incorporation de $10 \%$ de farines d'asticots séchés. Les poulets ayant consommé la ration R2 composé de $5 \%$ de farine d'asticots ont affiché un taux de mortalité moyen de $12 \%$. 
Aucune maladie ou lésion organique n'a été observée à l'autopsie. Les taux de mortalité ont connu une baisse remarquable chez tous les lots de poulets à l'issue de la période de croissance. Aucune mortalité n'a été enregistrée au cours de cette période.

\section{Consommation alimentaire des poulets de chair}

La consommation alimentaire des poulets de chair au cours des essais est présentée dans le Tableau 7. Il ressort de l'analyse du Tableau 7 que les poulets de chair ont consommé en moyenne par tête entre $35 \mathrm{~g}$ et $40 \mathrm{~g}$ de MS d'aliment pendant la première semaine d'essais. Cette consommation a progressé en moyenne de $20 \mathrm{~g}$ de MS chez tous les poulets pendant la deuxième semaine, indépendamment de la nature de l'aliment. Pendant la troisième semaine, la consommation alimentaire des poulets était de 73,6 g ; 76,3 g et de 75,10 $\mathrm{g}$ de MS respectivement pour les lots des rations R1, R2 et R3. La consommation alimentaire moyenne des poulets nourris avec la ration témoin $\mathrm{R} 1$ a quasiment doublé de la troisième à la quatrième en passant de $73.6 \mathrm{~g}$ à $143,50 \mathrm{~g}$ de MS, dépassant ainsi respectivement de $21 \%$ et de $20,3 \%(p<0,05)$ celle des poulets nourris avec les rations R2 et R3. La consommation alimentaire moyenne a augmenté de 22,03 g ; 64,3 g et 50,1 g de MS au cours de la cinquième ( $1^{\text {ère }}$ semaine de croissance) respectivement chez les poulets nourris avec les rations R1, R2 et R3., sans aucune différence significative $(p>0,05)$ entre les différents lots. A partir de la sixième semaine d'essai, les niveaux de consommation alimentaire se sont sensiblement rapprochés dans leur progression chez tous les lots d'animaux pour atteindre respectivement 307,4 $\mathrm{g} ; 327,7 \mathrm{~g}$ et $315,7 \mathrm{~g}$ de MS pour les lots R1, $\mathrm{R} 2$ et R3. Les rations R2 et R3 contenant la farine d'asticots séchés étaient en fin de croissance plus consommée comparativement à celle du lot R1. Bien qu'aucune différence significative $(p>0,05)$ n'ait été observée parmi les lots d'animaux pour la consommation des aliments, que ce soit pour le démarrage, qu'en fin de croissance, une meilleure utilisation des aliments a été constatée chez les poulets nourris d'aliments contenant de la farine d'asticots séchés.

\section{Evolution pondérale}

Au début de l'essai, les poulets avaient un poids moyen de $38 \mathrm{~g}$ et aucune différence significative $(p>0,05)$ n'a été observée entre les différents lots (Tableau 8). Il ressort de ce tableau qu'à la fin de la phase de démarrage ( $28^{\text {ème }} \mathrm{j}$ ), le lot témoin R1 a donné un poids de $225,5 \mathrm{~g}$ par poulet et ceux des lots R2 et R3 avaient respectivement un poids moyen de $245,10 \mathrm{~g}$ et $251,10 \mathrm{~g}$. On a observé une différence significative $(\mathrm{p}<0,05)$ de poids entre les différents lots de poulets. Les sujets recevant la farine d'asticots séchés étaient plus lourds que les témoins. Au $42^{\text {ème }}$ jour, le poids moyen des poulets étaient respectivement de $825,5 \mathrm{~g} ; 830 \mathrm{~g}$ et $858,5 \mathrm{~g}$ pour les lots $\mathrm{R} 1, \mathrm{R} 2$ et R3. On observe également une différence significative $(\mathrm{p}<0,05)$ de poids entre les différents lots de poulets. Cette différence de poids reste significative $(\mathrm{p}<0,05)$ jusqu'au $56^{\text {ème }}$ jour où les poulets de chair des lots R1, $\mathrm{R} 2$ et R3 pèsent respectivement 1325,5 $\mathrm{g}$; $1550,5 \mathrm{~g}$ et $1705,5 \mathrm{~g}$.

\section{Vitesse de croissance (gain moyen quotidien des poulets de chair)}

Le Tableau 9 présente le gain de poids des poulets au cours de la période d'expérimentation. Il ressort de l'analyse du tableau que les poulets du lot R1 (témoin) ont un gain moyen quotidien significativement $(\mathrm{p}<0,05)$ plus faible que ceux des poulets des lots R2 et R3 durant toutes les périodes d'essais. Le rythme de croissance quotidien a été en constante progression chez tous les poulets, quel qu'en soit la ration alimentaire. D'une manière générale, les poulets des lots R2 et R3 nourris avec de la farine d'asticots séchés ont présenté les meilleurs gains moyens quotidiens sur toutes les périodes d'essais. 
Tableau 5 : Composition chimique de la farine d'asticots séchés et du tourteau de soja.

\begin{tabular}{lcc}
\hline Nutriments $(\%$ MS) & Farine d'asticots séchés & Tourteau de soja \\
\hline Matière Sèche & 91,56 & 88,09 \\
Matières Azotées Totales & 59,65 & 51,52 \\
Matière Grasse & 22,25 & 01,98 \\
Cellulose Brute & - & 06,25 \\
Cendres Totales & 08,3 & 07,13 \\
Extractifs Non Azotés & 14,92 & 24,18 \\
Ca (g/kg) & 04,7 & 03,4 \\
P (g/kg) & 05,7 & 06,4 \\
Acide linolénique $(\% \mathrm{MG})$ & 02,0 & 01,7 \\
E.M. (Kcal/kg MS) & 4539 & 3500 \\
\hline
\end{tabular}

Source: laboratoire du Génie des Procédés.

Tableau 6 : Effectifs des poulets des différents lots en fonction de l'âge en jours.

\begin{tabular}{|c|c|c|c|c|c|c|}
\hline Lots & Age & $1^{\mathrm{er}}$ & $14^{\text {ème }}$ & $23^{\text {ème }}$ & $42^{\mathrm{ème}}$ & $56^{\text {ème }}$ \\
\hline$\overline{\mathrm{R} 1}$ & & 25 & 22 & 20 & 20 & 20 \\
\hline $\mathrm{R} 2$ & & 25 & 23 & 22 & 22 & 22 \\
\hline $\mathrm{R} 3$ & & 25 & 23 & 23 & 23 & 23 \\
\hline Total & & 75 & 68 & 65 & 65 & 65 \\
\hline
\end{tabular}

Tableau 7 : Consommation alimentaire moyenne (g de MS/tête/semaine) des poulets de chair au cours des essais.

\begin{tabular}{|c|c|c|c|c|c|}
\hline \multicolumn{2}{|c|}{ Périodes d'essais et types de rations } & \multicolumn{4}{|c|}{ Période de démarrage (semaines) } \\
\hline \multirow{4}{*}{ Rations de démarrage } & & 1 & 2 & 3 & 4 \\
\hline & $\mathrm{R} 1$ & 40 & 57,7 & 73,6 & 143,5 \\
\hline & $\mathrm{R} 2$ & 34,5 & 56,5 & 76,3 & 114,4 \\
\hline & R3 & 35,9 & 57,35 & 75,10 & 113,4 \\
\hline \multirow{5}{*}{ Ration de croissance } & & \multicolumn{4}{|c|}{ Période de croissance (semaines) } \\
\hline & & 5 & 6 & 7 & 8 \\
\hline & $\mathrm{R} 4$ & 165,6 & 213,2 & 279,8 & 307,4 \\
\hline & R5 & 178,7 & 224,0 & 296,10 & 327,7 \\
\hline & R6 & 163,3 & 223,0 & 277,90 & 315,70 \\
\hline
\end{tabular}

Tableau 8 : Poids vif des poulets de chair au cours de l'essai.

\begin{tabular}{lccccc}
\hline Age (jours) & $\mathbf{1}^{\text {er }}$ & $\mathbf{1 4}^{\text {ème }}$ & $\mathbf{2 8}^{\text {ème }}$ & $\mathbf{4 2}^{\text {ème }}$ & $\mathbf{5 6}^{\text {ème }}$ \\
Lots & & & & & \\
\hline R1 & 38 & 117,50 & 225,50 & 825,50 & 1325,50 \\
R2 & 38 & 110,60 & 245,10 & 830,00 & 1550,50 \\
R3 & 38 & 119,70 & 251,10 & 858,50 & 1705,50 \\
\hline
\end{tabular}


Tableau 9 : Gain moyen quotidien (g) des poulets de chair pendant les périodes de démarrage et de croissance.

\begin{tabular}{lccccc}
\hline Périodes d'essais et types de rations & \multicolumn{4}{c}{ Période de démarrage (semaines) } \\
\hline \multirow{2}{*}{ Rations de démarrage } & R1 & 1 & 2 & 3 & 4 \\
& $\mathrm{R} 2$ & 1,17 & 1,73 & 2,73 & 2,14 \\
& $\mathrm{R} 3$ & 1,70 & 1,89 & 3,87 & 2,45 \\
& & 5 & 6 & 7 & 8 \\
\multirow{2}{*}{ Rations de croissance } & $\mathrm{R} 4$ & 3,45 & 6,20 & 5,10 & 6,00 \\
& $\mathrm{R} 5$ & 3,50 & 6,50 & 5,55 & 8,85 \\
& $\mathrm{R} 6$ & 4,85 & 5,95 & 5,50 & 10,20 \\
\hline
\end{tabular}

\section{DISCUSSION}

La farine d'asticots séchés comme source de protéine et de matière grasse dans l'alimentation animale a fait l'objet de plusieurs études depuis plusieurs décennies (Bouafou, 2007). Cette étude confirme les teneurs particulièrement élevées en MAT $(91,56 \%)$ et en MG $(22,25 \%)$ de la farine d'asticots séchés. Ces taux protéiques et lipidiques plus élevés en ont fait une source alternative aux farines animales habituellement utilisées en élevage (Kande, 2012). L'utilisation partielle de la farine d'asticots séchés en substitution du tourteau de soja a permis de mesurer son importance alimentaire et nutritionnelle sur les performances zootechniques des poulets de chair. Le taux de $91,56 \%$ de MS obtenu dans la présente étude est comparable à celui de $92,5 \%$ obtenu par Bouafou (2007) sur des asticots vieux de deux jours séchés à l'étuve. Il en est de même du taux de 22,25\% de MG comparé à celui de $41,1 \%$ obtenu par Bouafou (2007) après 12 heures de séchage dans une étuve. Le taux de MAT de cette étude reste cependant inférieur à celui obtenu par Kouame (2008) pour qui, la composition chimique des asticots dépendrait de leur stade de développement larvaire et de la durée de séchage (Pomalegni, 2016). En outre, les résultats des travaux de Loa (2000) sur la production et l'utilisation contrôlée des asticots, a permis de montrer que les asticots constituent un aliment préférentiel des volailles par rapport aux céréales. Quant au tourteau de soja, il est une excellente source de protéines, d'énergie, d'acide linolénique, de lysine, de vitamine B, d'acides gras, de calcium et de phosphore (Carine, 2014).

$\mathrm{La}$ consommation alimentaire des poulets de chair est restée quasiment identique dans sa progression au cours des trois premières semaines, quel que soit le niveau d'incorporation de la farine d'asticots séchés dans la ration. La consommation d'aliments a été sensiblement supérieure chez les poulets nourris aux rations contenant de la farine d'asticots séchés comparée au lot témoin. En fin de croissance la consommation des rations des lots R2 et R3 était plus intéressante que celle de la ration du lot R1. Les niveaux de consommation des régimes contenant de la farine d'asticots séchés étaient comparables à celui d'un régime contenant de la farine de poisson rapportée par Watson (2006) puis Bouafou (2011). L'incorporation de la farine d'asticots séchés de l'ordre de $10 \%$ en substitution du tourteau de soja dans la ration R3 a considérablement limité l'ingestion alimentaire des poulets. Ce comportement alimentaire peut s'expliquer par la richesse énergétique de la ration favorisée par un taux particulièrement élevé de MG de la farine d'asticots séchés. En effet, les volailles avaient en général tendance à réguler leur ingestion alimentaire en fonction de la densité énergétique de la ration (Lessire, 1995). Nos résultats corroborent avec ceux obtenus par Lessire avec la farine de viande, la farine de poisson et le tourteau de soja qui sont des 
protéines habituellement utilisées dans l'alimentation du bétail, de la volaille ou des poissons. Cependant, la bibliographie indique qu'un régime riche en protéines animales contribue à l'augmentation de la calciurie qui est un facteur de risque de lithiase calcique ou de calculs rénaux (Bouafou, 2011). Des taux d'incorporation supérieurs à $11 \%$ entraînent généralement une diminution de la prise alimentaire et des performances des volailles qui pourrait être liée à la couleur foncée de l'aliment. Cette remarque peut suggérer que l'incorporation de la farine d'asticots séchés dans l'alimentation ne serait bénéfique qu'à un faible taux.

Le gain de poids à la période de démarrage et à celle de la fin de croissance a été élevé chez les sujets nourris avec la ration contenant de la farine d'asticots séchés comparé aux sujets témoins nourris avec la ration ne contenant que du tourteau de soja. En général, l'apport de nutriments provenant d'asticots au régime a occasionné un gain de poids important comparativement au lot témoin. Ceci corrobore avec les résultats des travaux de Loa (2000) sur la production et l'utilisation contrôlée d'asticots. Cette différence en gain de poids pourrait être liée à la qualité d'aliment mais aussi à l'environnement. Les meilleures performances obtenues avec la farine d'asticots séchés pourraient aussi s'expliquer par la proportion de cellulose plus élevée dans la ration R1 par rapport aux rations $\mathrm{R} 2$ et $\mathrm{R} 3$.

Le taux de mortalité était nettement significatif entre les du lot R1 et ceux du lot R3 durant la période de démarrage. La ration intermédiaire R2 constituée de 5\% de farine d'asticots séchés n'a pas permis de baisser significativement le taux de mortalité des poulets au cours de la phase de démarrage. A l'issue de la phase de croissance, les taux de mortalité au sein de tous les lots d'animaux ont connu une baisse sensible. Tous les poulets de chair étaient devenus moins vulnérable à cet âge.

\section{Conclusion}

$\mathrm{Au}$ terme de cette étude qui s'est assignée comme objectifs d'analyser et d'évaluer les effets d'une substitution partielle du tourteau de soja par la farine d'asticots séchés dans des rations alimentaires sur les performances zootechniques des poulets de chair, il en ressort que l'utilisation de la farine d'asticots séchés comme source de protéine animale s'est révélée envisageable dans l'alimentation des poulets de chair. La substitution de $10 \%$ dans la ration a permis d'augmenter substantiellement les teneurs en MAT et en MG. L'amélioration qualitative de la ration a permis de réduire le taux de mortalité des poulets tout en améliorant l'indice de consommation à travers une baisse significative de la consommation alimentaire. Une substitution de $5 \%$ du tourteau de soja par la farine d'asticots séchés dans la ration a été certes amélioratrice mais pas assez pour induire une différence significative. L'utilisation du tourteau de soja et de la farine d'asticots séchés semble optimale à faible taux et peut mieux être recommandée en aviculture. Le taux d'incorporation de $10 \%$ dans la ration n'ayant entraîné aucune mortalité chez les poulets de chair, l'étude va continuer avec d'autres taux d'incorporation afin de déterminer la limite maximale d'incorporation de farine d'asticots, en vue de la mise au point des formules alimentaires efficaces et efficientes pour la volaille.

\section{CONFLITS D'INTERETS} d'intérêts.

Les auteurs ne déclarent aucun conflit

\section{CONTRIBUTIONS DES AUTEURS}

Tous les auteurs ont contribué à la prise en charge du matériel didactique et à la rédaction du manuscrit. Tous les auteurs ont lu et approuvé la version finale du manuscrit.

\section{REMERCIEMENTS}

Les auteurs expriment leurs sincères remerciements aux vétérinaires, éleveurs de la localité de Dang pour leur aide précieuse sur le terrain et en particulier à Mrs. Avoungoe et 
Dawaga, pour leur apport dans la construction du bâtiment où ont eu lieu les travaux.

\section{REFERENCES}

Atteh JO. Ologbenla FD.1993. Replacement of fish meal with maggots in broiler diets: effects on performance and nutrient retention. Nigerian Journal of Animal Production 20 (1): 44-49. DOI: 10.51791/njap.v20i.2100

Bouafou KGM. 2011. Revue bibliographique sur les asticots et leur emploi dans l'alimentation animale. J. Anim. Plant. Sci., 12 (2): 1543-1551.

Bouafou KGM. Kouamé MG. 2007. Bilan azoté chez le rat en croissance de la farine d'asticots échés. Tropicultura, 25 (2) : 7074.

Carine C. Hedji. Diane N. Emile D. 2014. Valorisation de Azolla spp, Moringa oleifera, son de riz et de co-produitsde volaille et de poisson en alimentation animale : J. Appl. Biosci., 81 : 7277-7289. DOI : $10.4314 / J A B . V 8111.4$

Kande. 2012. Contribution à l'étude des substrats adéquats pour la production d'asticots comme aliment pour les animaux monogastriques à Kinshasa : Fascicule de TPE en Zootechnie.Faculté des Sciences Agronomiques, Unikin, 29p

Kenis M. Kone C. 2014. Insects used for animal feed in West Africa.Entomologia, 2 (2): 107- $114 . \quad$ DOI : 10.4081/entomologia.2014.218.

Kouame GM. 2008. Étude de la valeur nutritionnelle de la farine d'asticots séchés chez les rats en croissance. Rev. Ivoir. Sci. Technol., 12: 215-225.

Lessire M. Revol N. Rudeaux F. Hallouis JM.1995. Valeur énergétique des aliments chez la poule pondeuse. INRA Prod.
Anim., $\quad$ 8(3) : $\quad 189-195 . \quad$ DOI : $10.2087 /$ productionsanimales.1995.8.3.4127.

Loa C. 2000. Production et utilisation contrôlée d'asticots. Tropicultura, 18 (4) : 215-219.

Mangunga E. 2013. Synthèse des travaux de recherches sur la production d'asticots pour l'alimentation des monogastriques : Cas de la volaille et des rongeurs, $29 \mathrm{p}$.

Minepat. 2013. Croissance démographique du Cameroun de 1960 à 2013 : Indicateurs clés de l'économie camerounaise, 8p.

Mpoame M. Teguia A. Nguemfo E. 2004.Essai comparé de production d'asticots dans les fientes de poule et dans la bouse de vache. Tropicultura, 22 (2) : 84-87.

Pomalegni SC. Edenakpo KA. Ahoyo NR. 2020. Perception, performances zootechniques et qualité nutritionnelle de la viande de poulets locaux (Gallus gallus) nourris avec des rations alimentaires à base de larves de mouche (Musca domestica Linnaeus1758) au Benin. Agronomie Africaine, 32 (1) : 1524.

Pomalegni SC. Gbemavo D. 2016. Synthèse bibliographique sur les insectes et autres invertébrés comestibles utilisés dans l'alimentation des animaux monogastriques d'élevage. Bulletin de la Recherche Agronomique du Benin, 184 (80):43-53.

Teguia A. Mpoame M. Okourou JA. 2002. The production performance of broiler birds as affected by the replacement of fish meal by maggot meal in the stater and finisher diets. Tropicultura, 20 (4): 187-192.

Watson H. 2006. Poultry meal vs poultry byproduct meal. Dogs in Canada Magazine, January 2006, 2p. 